tinted glasses, suggested by Dr. Murrell, but can readily understand that it must give great additional relief in these alpinotic eyes on bright days. I was greatly interested at the time in the old lady, an albino, with mature hard cataract in both eyes, to whom Dr. Gould has kindly alluded in hls paper. The lens was successfully extracted in one eye. Vision 6-12 was secured with glasses $+1.50 \mathrm{D}$. There was a large posterior staphyloma at the temporal side of the nerve, so that this patient had evidently been myopic before the occurrence of the cataract.

Dr. Gour.D-I would like to ask the gentlemen when they have such cases to please send me a report of them.

\section{TUMOR OF THE IRIS.}

Read in the Section on Ophthalmology, at the Forty-fourth Annual Meeting of the American Medical Association.

BY J. SCHNEIDER, M.D.

MILWAUKEE, WIS.

Tumors of the iris not caused by infection, such as syphilis or tuberculosis are very rare in our clinical experience, and when seen the majority have arrived at that stage of development when the integrity of the eye has been so changed that in order to arrest farther and more serious consequences there remains but one alternative, that of enucleation. In the literature on the subject I have not been able to find a similar case, and in my personal experience but this one, where after the removal of such a tumor from the iris there has remained usefulvision; and in the light of these facts I consider the following case very unique in its clinical and anatomical results :

Miss M., of Iron Mountain, Mich., consulted me with reference to a disturbance in the vision of her left eje. On awaking the morning of the 29 th of March, 1892, she noticed the sight of the eye much reduced and objects seen with it appeared a dark red color. On March 30,1892 , the patient came to me, when I found the following conditions: R. E., normal; L. E., ability to distinguish the movement of a hand at ten feet; peripheral perception of light normal; no pain; no inflammatory symptoms. The anterior chamber was filled with blood; external layer of the cornea normal, but the lower temporal quadrant of the middle and internal layers were hazy, the haziness extending four or five $\mathrm{mm}$. into the cornea; tension somewhat increased.

After ten days' treatment the blood in the anterior chamber had disappeared, at which time I was able to make an examination of the deeper structures. The vision was $20-30$; colors and field of vision normal. In the lower temporal quadrant of the anterior chamber was lying a mass partially filling in the space between the iris and cornea, but touching the cornea near the corneo-sclero junction only, thus leaving a shallow anterior chamber above the growth. It could plainly be seen that the neoplasm had its origin in the ligamentum pectinatum iridis, and extending nearly to the sphincter-pupillaris, filling almost completely the lower temporal quadrant. In consistence it appeared medullary, reddish yellow color, shaped similar to a half coffee bean with the flat side lying directly upon the iris. The portion of the growth nearest the corneo-scleral junetion being pressed upon by the cornea was flattened and grayish white in appearance: the remainder appearing very vascular and studded with papillæ. With a light reflected directly upon the growth it could plainly be seen that the tumor grew directly out from the iris. The longest diame. ter was $9 \mathrm{~mm}$., the shortest $5 \mathrm{~mm}$. The crystalline lens and vitreous were apparently normal; reaction of pupil to light, and appearance of iris as far as the periphery of the tumor were normal. The outline of the optic disc in the lower temporal quadrant was blurred; this cloudiness, for the distance of a disc diameter, extending into the retina and finally blending by means of white streaks into the normal tissue of the retina.

The patient was 16 years of age, menstruated regularly since her twelfth year, rather anemic, had never had any severe illness, body well nourished, family history tubercu. lous, but repeated examinations for symptoms of phthisis and syphilis always giving a negative result.
The parents state that at the age of 5 years the girl was injured in the left eye with a fork; five months later they noticed a small brown spot near the pupil. This spot did not change till four years ago when with no apparent cause it assumed a flesh color and began to increase in size. After several examinations I recommended, especially on account of the family history, that the growth be removed, but it was not agreed to by the parents and for some time the patient passed away from observation.

On March 24, 1893, the patient returned, complaining of a dull pain in the eye, tearing, sensitiveness to light with recurrent hemorrhages (the patient could produce a hemorrhage at will by pressure upon the growth). During the period of her absence the neoplasm had grown in all directions, extending upwards so as to cover one-third of the pupil; tension increased, cornea hazy and vision reduced to less than one-half of the normal. The optic disc and retina showed marked symptoms of pressure atrophy. We have to take into consideration that we have before us a solid, flesh-colored tumor, very vascular and probably not the product of a general dyscrasia. The tumor is definitely located, non-pigmented, the surrounding parts, i.e., the iris showing no pathological changes.

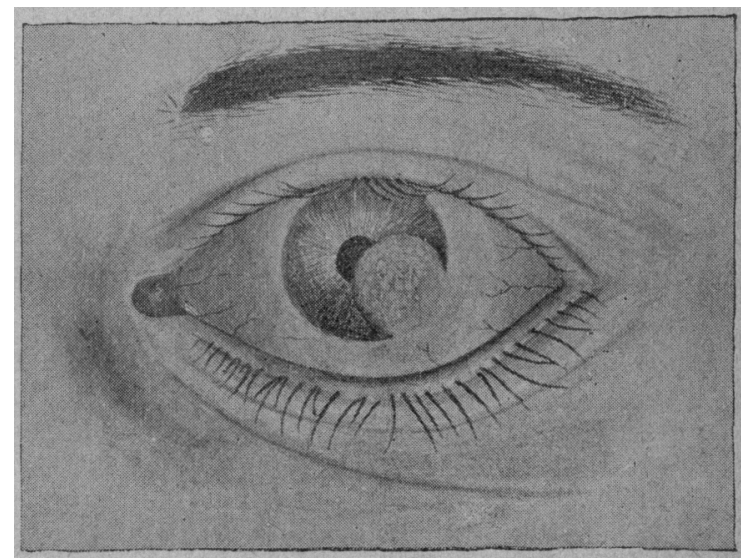

On account of the family history I was inclined to consider it a solitary tubercle or so-called granuloma. The negative results of the physical examinations would not be positive proof against a tubercle, for we know from Gradenigo, Perls, Haab, Cohnheim, Knapp and others that tubercular degeneration can be localized in the iris without any constitutional symptoms. Pudden, Haab and others affirm that granuloma have for their origin the irritating effects of some microörganism. Second, granuloma are poorly organized neoplasms, infectious, inoculable and invade neighboring tissues, especially those with which they are in direct contact. The granuloma undergoes, on account of its poor vascularization, cloudy swelling or fatty degeneration. Aside from these authorities, the clinical appearance of the neoplasm made me doubt the existence of a tubercle. I was inclined to consider it a non-pigmented sarcoma, but the age of the patient and the clinical appearance of the growth were evidences against a sarcoma. We can immediately exclude all neoplasms of the iris such as gumma, epidermoidome cyst, cystoid degeneration and melano-sarcoma.

On March 28, 1893, the growth was removed in the 
following manner: after the eye was thoroughly cocainized, at a distance of $1 \frac{1}{2} \mathrm{~mm}$. from the corneosclero junction I made a linear incision, passing partially through the base of the tumor. With an iris forceps $I$ attempted to seize the mass but it being so fragile, only small particles could be removed; with a platinum loop I made similar attempts with the same results, it being very evident that the iris was attached to the capsule of the lens. With an iris forceps armed with a double row of teeth I seized the iris on either side of the tumor and with some little effort withdrew the iarge mass and attached portion of the iris out of the anterior chamber, which mass was then excised as close as possible to the base. (There still remained some parts of the growth broken off by the manipulations, in the anterior chamber.) After the growth and the attached portion of the iris were excised there followed a severe hemorrhage. After the hemorrhage had ceased and the lips of the wound were adjusted, I applied a pressure bandage and put the patient to bed. During the healing process the eye showed but very little reaction and on April 23, 1893 the patient was discharged with tension normal and, with the exception of a small zone, the former corneal haziness had disappeared, some pigmented spots upon the capsule in the coloboma, lower temporal quadrant of disc pale, retina changes disappeared and vision 20-30.

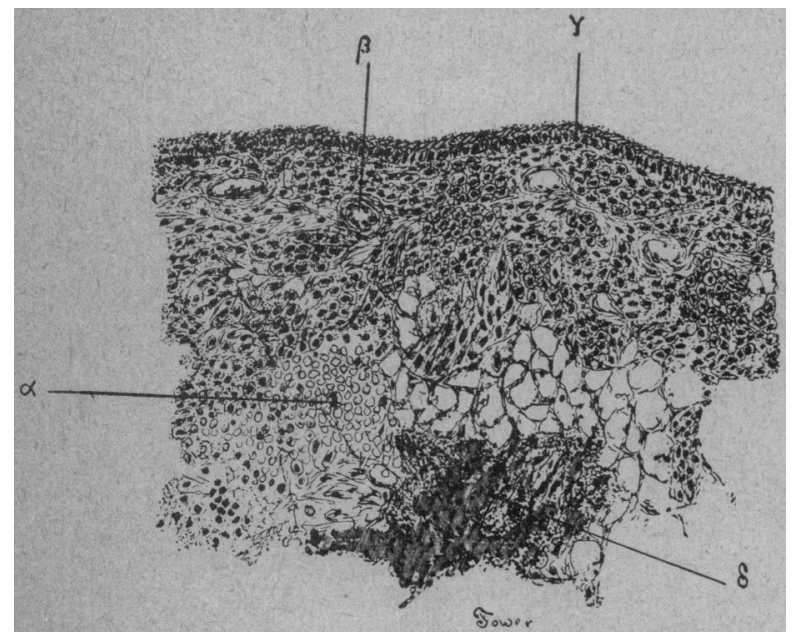

DESCRIPTION OF PLATE.

A. Small hemorrhage in growth just above iris in loose connective tissue showing very plainly the biood corpuscles.

B. Blood vessel. One of many which are seen scattered throughout and containing blood corpuscles.

covering cells.

D. Portion of iris from which the tumor probably originated.

The tumor measured $7 \mathrm{~mm}$. in its longest diameter, $5 \mathrm{~mm}$. in its shortest and $3 \mathrm{~mm}$. in thickness; its superficial surface was uneven grayish-white, with the base showing normal appearance of iris. After its removal the tumor was placed in a sodium-chlorid solution, and shortly afterwards given to Dr. Tower of Milwaukee, for microscopical examination, who reports as follows:

"I took small particles, every precaution being taken to prevent them from being contaminated and planted them in tubes of agar, blood-serum, gelatin, and in bouillon both acid and alkalin, and kept them at 37 degrees centigrade and also at about 20 degrees centigrade for several days, and was unable to get any growths except in one tube a little penicellium which was a contamination from without, either from the air or some of the utensils used. I examined stained preparations for the tubercle bacilli with negative results; in fact, did not find any germs at all or evidence of them.

"I have made examinations directly of small portions when fresh, under the microscope, and found a connective tissue and epithelial growth from apparently the iris. Upon section and examination, I find the growth to be a papilloma originating from the sub-epithelial connective tissue of the iris."

We understand by a papilloma, a neoplasm composed of connective tissue with epithelial covering; with very complete vascularization, resembling in construction skin, intestinal and mucous membrane papillæ (Wagner). Papilloma start usually from an injury, producing increased circulation to the part, causing a similar irritation to that produced by dirt in the formation of warts and by decomposing glandular secretions in the formation of condylomæ. Such a papilloma may originate in any tissue as they are found in paroöphoron, cystic tumors of broad ligament (Mouillin), the skin, tongue, larynx, conjunctiva, fore-skin, glans penis, rectum, synovia! membranes. If the tumor under consideration was of leprous, tuberculous or actini-mycotic growth, the germs characteristic of these growths would have been found. If it had been any of these, or a syphilitic growth or a rhino-sclerotic, the particles remaining in the anterior chamber would have developed into a new growth.

I consider the prognosis in this case very favorable; especially on account of the age of the patient if there were particles remaining there would be no probability of the formation of a villous carcinoma, into which the papilloma sometimes develop.

\section{THE CLASSIFICATION OF DISEASES OF THE EYE FOR HOSPITAL STATISTICS.}

Read in the Section on Ophthalmology at the Forty-fourth Annual Meeting of the American Medical Association.

BY EDWARD JACKSON, A.M., M.D.

PROFESSOR OF DISEASES OF THE FYE IN THE PHILADELPHIA POLYCLINIC; SURGEON TO WILLS EYE HOSPITAL.

The custom has become general among hospitals and dispensaries, of publishing Annual Reports, showing the number of cases treated. The principal value of such statistics, from the scientific point of view, is that they may throw light on the etiology of certain diseases, by showing their comparative frequency in different places and at different times. This can only attach to such statistics when the classification they employ is comparatively uniform, and their value, as well as the ease with which they can be handled in combination, will be enormously increased if the classifications be exactly uniform.

On consulting the reports of the principal ophthalmic hospitals of the country, it will be found that in the main they pretty closely agree as to the naming and relative frequency of the mass of conditions mentioned. There are, however, points of divergence which render the comparison of statistics obtained in the different large cities difficult and often valuless.

To elicit discussion, as well as to urge their importance, certain characteristics of a good classification may be mentioned.

First, it must not be so minute as to be seriously burdensome. With the future development of ophthalmic science, it will be possible, perhaps, to bring 